\title{
El género Apiococcus Hempel (Hemiptera, Eriococcidae), con redescripción de dos especies
}

\author{
Patricia González ${ }^{1} \&$ Lucía E. Claps ${ }^{1}$
} 'Instituto Superior de Entomología “Dr. Abraham Willink” (INSUE), Facultad de Ciencias Naturales e Instituto Miguel Lillo, Universidad Nacional de
Tucumán. Miguel Lillo 205. 4000, Tucumán, Argentina. mopagon2004@yahoo.com.ar; luciaclaps@arnet.com.ar

\begin{abstract}
The genus Apiococcus Hempel (Hemiptera, Eriococcidae), with redescription of two species. Apiococcus Hempel is a genus from Brazil composed of four gall-inducing species. The adult females of two species, Apiococcus globosus Hempel and $A$. singularis Hempel, from Brazil, are redescribed and illustrated. Keys to the species of the genus and their galls are given.
\end{abstract}

KEYWORDS. Insecta; Neotropical; Brazil; Myrtaceae; galls.

Dentro de la familia Eriococcidae (Coccoidea) hay varios géneros formadores de agallas, poco conocidos en Sudamérica (Cockerell, 1899; Hempel, 1900 y 1934; Lepage, 1941; Ferris, 1957a y b; Silva d'Araujo et al., 1968; Miller \& Gimpel 2000; Hodgson et al. 2004). Uno de ellos, Apiococcus Hempel, citado sólo en Brasil, contiene cuatro especies que tienen a especies de la familia Myrtaceae como plantas hospederas. Este género fue redescripto recientemente, al igual que la especie tipo Apiococcus gregarius Hempel, por Hodgson \& Miller (2010). Ya que las descripciones de Hempel de las otras tres especies son incompletas y el material tipo de Apiococcus asperatus Hempel no está en buenas condiciones, este trabajo tuvo por objetivo redescribir e ilustrar la hembra adulta de dos especies, Apiococcus globosus Hempel y A. singularis Hempel y presentar una clave dicotómica para las cuatro especies del género y de sus agallas.

\section{MATERIAL Y MÉTODOS}

El material estudiado proviene de la colección del $\mathrm{Mu}-$ seu de Zoología, Universidad de São Paulo, Brasil (MZSP). Se observaron preparaciones microscópicas y cubiertas externas conservadas en seco en tubos de vidrio rotuladas como tipo, las que fueron fotografiadas empleando cámara digital de 4,0 megapíxeles.

El material tipo de Apiococcus asperatus se encuentra en malas condiciones; se intentó realizar preparaciones microscópicas nuevas, empleando el material seco depositado en la misma colección. Luego de la hidratación se siguió una de las técnicas empleadas comúnmente para "cochinillas"; usando solución Essig (Granara de Willink 1990) e hidróxido de potasio al 10\% (Claps 1991) pero no fue posible realizar buenos preparados. Dada la imposibilidad de realizar las preparaciones microscópicas y a fin de no perder todo el material original seco, se decidió mantener el mismo y tomar de Hempel (1900) los caracteres de esta especie, usados en la clave. Los dibujos representan en la mitad derecha la superficie ventral y en la mitad izquierda la superficie dorsal; los detalles de algunas estructuras alrededor del dibujo central no guardan proporción entre sí. Se empleó un tubo de cámara clara de microscopio óptico, de distintos aumentos. Las medidas se expresan en milímetros $(\mathrm{mm})$ y en micrómetros (ìm), corresponden a las del lectotipo seguido, entre paréntesis, por las de los paralectotipos. La terminología usada sigue a Hempel (1900) y Hodgson \& Miller (2010).

\section{RESULTADOS}

\section{Apiococcus Hempel}

Apiococcus Hempel, 1900: 401. Especie tipo Apiococcus gregarius Hempel.

Diagnosis enmendada (modificada de Hodgson \& Miller 2010). Forma del cuerpo globosa. Dorso: dermis con o sin areolaciones. Setas de dos tipos, setas cónicas redondeadas, grandes, forman líneas por segmento, setas más angostas en segmentos abdominales posteriores. Macroconductos ausentes. Microconductos con apariencia bilocular frecuentes. Poros quinqueloculares en bandas en segmentos abdominales posteriores y en un grupo grande en la zona cefálica. Lóbulos anales poco desarrollados, con un grupo de setas; con una invaginación o bolsa con poros loculares en su interior, posiblemente asociados a los lóbulos anales. Placa media ausente. Anillo anal representado por una abertura membranosa. Margen no definido. Superficie ventral con setas laterales cónicas similares a las dorsales; setas setosas en segmentos abdominales posteriores. Macroconductos ausentes. Microconductos similares a los dorsales. Poros quinqueloculares en bandas en segmentos abdominales, dispersos en la superficie. Poros cruciformes ausentes. Antenas uni o bisegmentadas. Lóbulos frontales y tubérculos antenales ausentes. Labium con número desconocido de segmentos. Patas ausentes. Vulva en segmento VII. 
Nota. Coincidimos en los caracteres usados por Hodgson \& Miller (2010) para diagnosticar al género Apiococcus, sólo diferimos en: 1) dermis con areolaciones, no siempre, $A$. globosus presenta la dermis lisa, sin areolaciones, las restantes especies si las presentan; 2) antenas unisegmentadas, $A$. globosus tiene antenas bisegmentadas, en ello coincidimos con Hempel (1900) y 3) vulva no detectada, A globosus y $A$. singularis presentan vulva en el segmento VII.

\section{Clave para separar las agallas causadas por cuatro especies de Apiococcus}

1. Cubierta externamente de color negro .. 2

1 '. Cubierta externamente de otro color ..... 3

2. Cubierta con superficie externa áspera, con tubérculos .. A. asperatus

2'. Cubierta con superficie externa lisa ........... A. singularis

3. Cubierta de color castaño oscuro ................... gregarius

3'. Cubierta blanca con tinte color crema ........... . globosus

\section{Clave para especies del género Apiococcus}

1. Lóbulos anales no distinguibles, superficie del cuerpo no areolada A. globosus

1 '. Lóbulos anales distinguibles, no esclerosados, superficie del cuerpo areolada .. 2

2. Abertura anal rodeada por 16 setas setosas, lóbulos anales con seis setas . A. singularis

2'. Abertura anal rodeada por aproximadamente 30 setas setosas, lóbulos anales con mayor número de setas ...... 3

3. Lóbulos anales con 12-15 setas .................... gregarius 3'. Lóbulos anales con 8-10 setas A. asperatus

\section{Apiococcus gregarius Hempel}

Apiococcus gregarius Hempel, 1900: 402-403.

Material tipo examinado. En una etiqueta se lee: Apiococcus gregarius sp. nov. Type 146. Hembra. 3543 En otra etiqueta dice Apiococcus gregarius Hempel. N 95.109 (cuatro hembras adultas en regular condición), MZSP. Ypiranga (Brasil) en renovales de Myrtaceae (Hempel 1900). A fin de mantener la estabilidad de la especie se designa Lectotipo a la hembra de ubicación superior izquierda (marcada con un círculo) y Paralectotipos, a las tres restantes.

Diagnosis. Ano rodeado por aproximadamente 30 setas setosas. Lóbulos anales no esclerosados, con 12-15 setas, una de mayor tamaño.

Observaciones. Los caracteres métricos del Lectotipo y el rango de los paralectotipos revisados acá e indicados entre corchetes, difieren de la redescripción realizada por Hodgson \& Miller (2010) en: i) Longitud total 0,75-1,33 mm [1,55 (1,6$2,1) \mathrm{mm}$; ii) ancho total $0,7-1,35 \mathrm{~mm}[1,44(1,5-2,1) \mathrm{mm}]$; iii) setas dorsales cónicas y redondeadas de $4-5 \mu \mathrm{m}$ de largo y $5 \mu \mathrm{m}$ de ancho [9-12 $\mu \mathrm{m}$ de largo y 9-12 $\mu \mathrm{m}$ ancho, igual rango en paralectotipos); iv) setas ventrales setosas de $3-5 \mu \mathrm{m}$ de longitud [9-12 $\mu \mathrm{m}$, igual rango en paralectotipos]; v) an- cho de la abertura anal 6-7 $\mu \mathrm{m}$ [12 $\mu \mathrm{m}$, igual en paralectotipos]; vi) seta apical de 30-60 $\mu \mathrm{m}[72(62-81) \mu \mathrm{m}]$; vii) lóbulos anales con setas espiniformes de 13-22 $\mu \mathrm{m}[10-14$ (7-17) $\mu \mathrm{m}]$; viii) ancho del peritrema 15-17 $\mu \mathrm{m}$ [22 (26-31) $\mu \mathrm{m}]$; ix) longitud de clípeo-labro 160-170 $\mu \mathrm{m}$ [227 (231-235) $\mu \mathrm{m}]$ у x) 19-27 setas en lóbulos anales [10 (10-15) setas].

Aspecto externo (Fig. 3). La hembra forma una cubierta esférica dura, externamente de color castaño oscuro, interiormente lisa y con secreción cerosa blanca; con un orificio pequeño de forma esférica (Hempel 1900).

\section{Apiococcus asperatus Hempel} (Fig. 4)

Apiococcus asperatus Hempel, 1900: 404.

Material tipo examinado. Apiococcus asperatus sp. nov. Type 147. Hembra. 315. (cuatro hembras adultas en muy malas condiciones) MZSP. Ypiranga (Brasil) sobre renovales de Myrtaceae (Hempel 1900). No se designó lectotipo debido a que el material estudiado no se encontraba en buen estado y tampoco se consiguió realizar preparaciones microscópicas, a partir del material seco, tal como se indicó en la sección Materiales y Métodos.

Observaciones: si bien el estado de los preparados no permite su redescripción, sí se observa claramente la superficie con abundantes areolaciones y la zona anal, en uno de los ejemplares.

Aspecto externo (Fig. 4). La hembra forma una cubierta esférica dura, gruesa, negra y áspera exteriormente debido a unos pequeños tubérculos; la superficie interna es lisa y cubierta de una secreción blanca (Hempel 1900).

\section{Apiococcus globosus Hempel}

(Figs. 1 y 5)

Apiococcus globosus Hempel, 1900: 405.

Material tipo examinado. En una etiqueta se lee: Apiococcus globosus sp. nov. Type 148. Hembra. 3543. En otra etiqueta dice Apiococcus globosus Hempel. $\mathrm{N}^{\circ} 95.111$ (una hembra adulta en buena condición y una ninfa del segundo estadio) MZSP. São Paulo (Brasil) en la corteza de un arbusto de Myrtaceae (Hempel 1900). A fin de mantener la estabilidad de la especie se designa Lectotipo a la hembra adulta de la preparación microscópica.

Diagnosis. Ano protegido por cuatro setas setosas, una en cada extremo y rodeado por 12 setas. Lóbulos anales no desarrollados. Poros quinqueloculares abundantes en ambas superficies.

Redescripción. Cuerpo globoso. Longitud 1,44 mm; ancho $1,29 \mathrm{~mm}$.

Superficie dorsal. Dermis membranosa, lisa, con estriaciones en los segmentos abdominales. Setas dorsales de dos tipos, unas cónicas, grandes y redondeadas, de 7-12 $\mu \mathrm{m}$ de longitud y $5-10 \mu \mathrm{m}$ de ancho, numerosas en toda la superficie, dispuestas en líneas transversas por segmento abdominal, sin orden aparente en tórax, escasas en la región cefálica; setas más pequeñas de $5 \mu \mathrm{m}$ de longitud y $5 \mu \mathrm{m}$ de ancho en áreas mediolaterales del mesotórax y metatórax. Un segundo tipo 


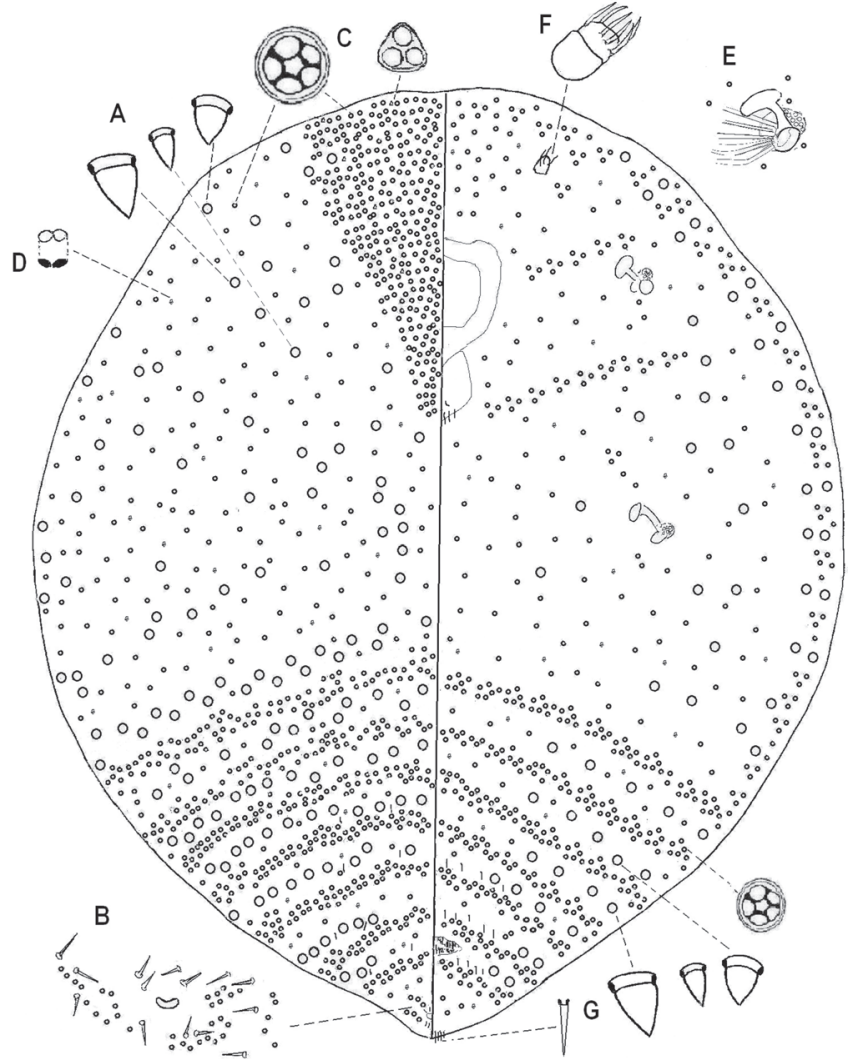

Fig. 1. Apiococcus globosus Hempel. Hembra adulta. A: setas dorsales, B: área anal, C: poros quinqueloculares y triloculares, D: microconducto, E: espiráculo, F: antena, G: setas ventrales.

de setas más angostas y con extremo agudo, de $12 \mu \mathrm{m}$ de longitud, en los segmentos abdominales posteriores, intercaladas con las setas redondeadas. Macroconductos ausentes. Microconductos aparentemente biloculares, de 1,5 $\mu \mathrm{m}$ de ancho y $2,5 \mu \mathrm{m}$ de longitud, dispersos en la superficie. Poros principalmente quinqueloculares de $5 \mu \mathrm{m}$ de diámetro y algunos triloculares, concentrados en bandas transversales a lo ancho de los siete segmentos preanales y en un grupo grande mediolateral, de aproximadamente $40 \mu \mathrm{m}$ de longitud y ancho (casi una cuarta parte de la longitud del cuerpo), en la región cefálica, abundantes, distribuidos regularmente en el resto de la superficie. Lóbulos anales no desarrollados. Anillo anal y setas del anillo anal ausentes. Ano representado por una abertura membranosa de $16 \mu \mathrm{m}$ de ancho, protegida por cuatro setas de 12-14 $\mu \mathrm{m}$ de longitud, una en cada extremo y rodeada por 10-12 setas de 12-14 $\mu \mathrm{m}$ de longitud cada una. Seta suranal no distinguible. Dos grupos no bien definidos de aproximadamente 20-25 poros en el área anal, corresponderían a dos invaginaciones alargadas o bolsas, características del género.

Margen. No definido.

Superficie ventral. Dermis membranosa. Setas de dos tipos, setas setosas de $7 \mu \mathrm{m}$ de longitud, con un total de 10-12 setas por segmento abdominal posterior; ocho setas de $12-$ $14 \mu \mathrm{m}$ de longitud en el extremo posterior del segmento VIII. Setas cónicas redondeadas similares a las del dorso, de 7-12 $\mu \mathrm{m}$ de longitud y $5-10 \mu \mathrm{m}$ de ancho, marginales y submar- ginales en la región cefálica y dos últimos segmentos abdominales, también mediolaterales en los segmentos II al $\mathrm{V}$ del abdomen y en tórax. Macroconductos ausentes. Microconductos de $1,5 \mu \mathrm{m}$ de ancho y $2,5 \mu \mathrm{m}$ de longitud, dispersos en la superficie. Poros quinqueloculares de $2,5 \mu \mathrm{m}$ de diámetro, concentrados en bandas transversas por segmento abdominal y entre los dos pares de espiráculos, abundantes distribuidos en el resto de la superficie. Espiráculos con concavidad pequeña en el extremo exterior, cada una con aproximadamente 15-20 poros; del extremo interior parten numerosas tráqueas formando radios. Antenas pequeñas, de dos segmentos, de $29 \mu \mathrm{m}$ de longitud, con seis setas terminales. Clípeo-labro de $287 \mu \mathrm{m}$ de longitud. Labium de $128 \mu \mathrm{m}$ de longitud, con cuatro pares de setas y número desconocido de segmentos. Ancho de peritrema de espiráculos $36 \mu \mathrm{m}$. Patas ausentes. Vulva en segmento VII.

Aspecto externo (Fig. 5). La hembra forma una cubierta esférica lisa exterior e interiormente, de color blanco con un tinte color crema; con un orificio pequeño de forma esférica (Hempel 1900).

\section{Apiococcus singularis Hempel}

(Figs. 2 y 6 )

Apiococcus singularis Hempel, 1900: 403.

Material tipo examinado. En una etiqueta se lee:Apiococcus singularis sp. nov. Type 182. Hembra. 19. En otra etiqueta dice Apiococcus singularis Hempel. $N^{\circ} 95.001$ (dos hembras adultas en regular condición) MZSP. Ypiranga (Brasil) sobre renovales de un arbusto de Myrtaceae (Hempel 1900). A fin de mantener la estabilidad de la especie se designa Lectotipo a la hembra ubicada en posición superior (marcada con un círculo) y Paralectotipo a la hembra restante.

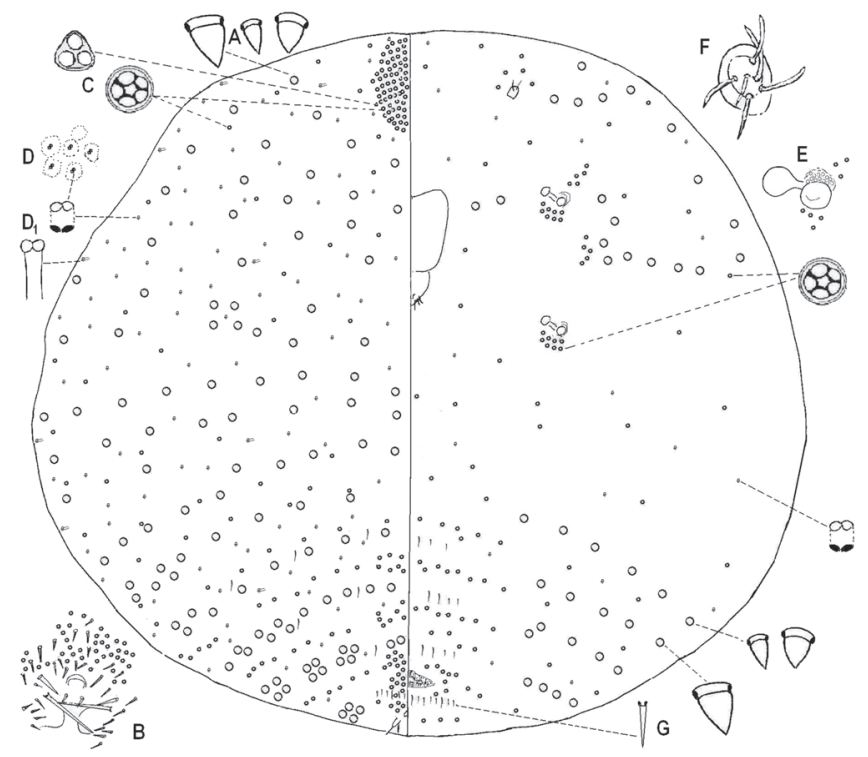

Fig. 2. Apiococcus singularis Hempel. Hembra adulta. A: setas dorsales, B: área anal, C: poros quinqueloculares y triloculares, D: superficie areolada mostrando la ubicación de los microconductos, D 1: microconductos de distinta longitud, E: espiráculo, F: antena, G: setas ventrales. 

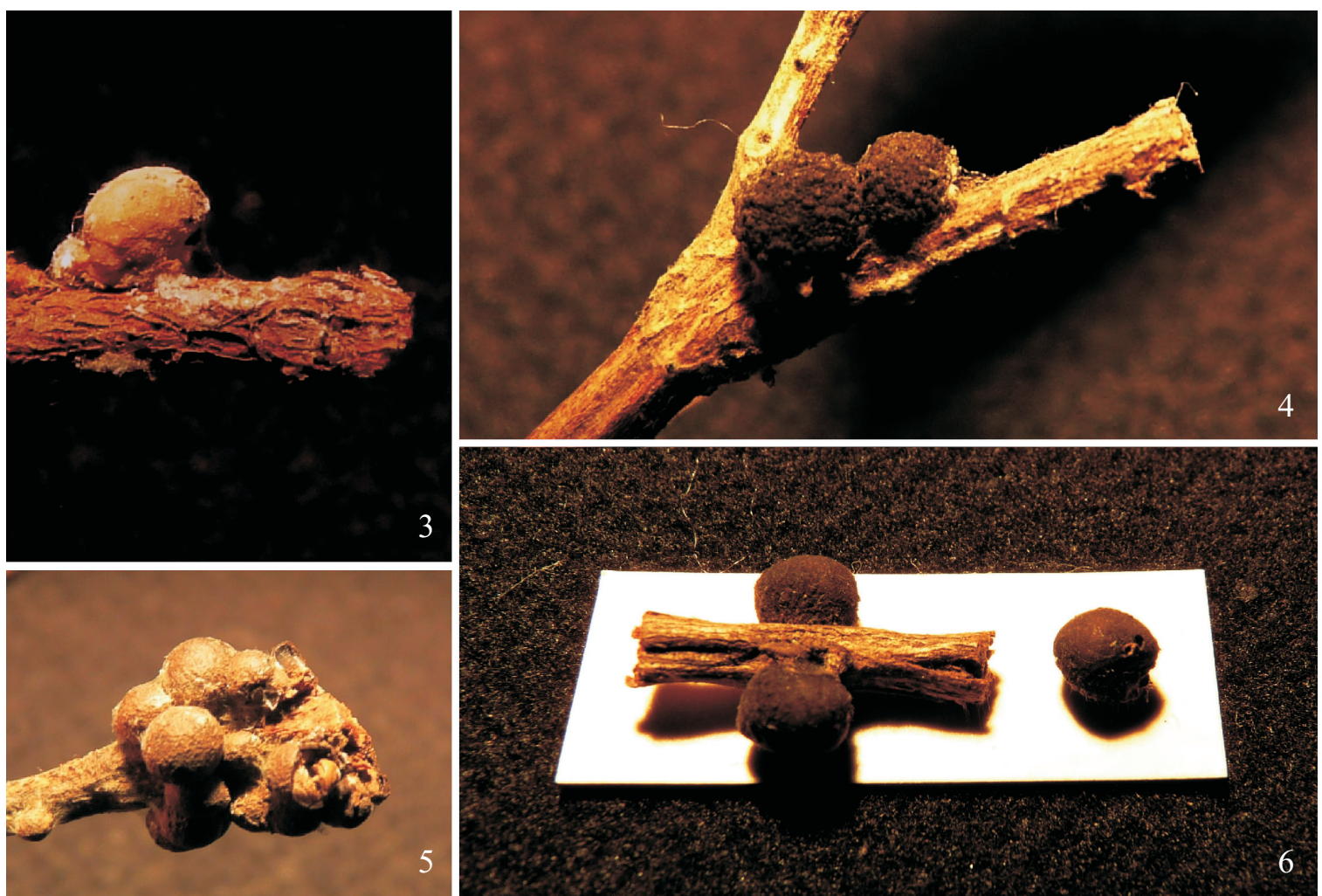

Figs. 3-6. Cubierta externa: 3. Apiococcus gregarius Hempel; 4. Apiococcus asperatus Hempel; 5. Apiococcus globosus Hempel; 6. Apiococcus singularis Hempel.

Diagnosis. Ano rodeado por 14 setas, dos de ellas de mayor tamaño. Lóbulos anales no esclerosados, con seis setas. Microconductos numerosos en la superficie dorsal.

Redescripción. Cuerpo globoso. Longitud 4,04 $(3,90)$ $\mathrm{mm}$; ancho 3,40 $(4,35) \mathrm{mm}$.

Superficie dorsal. Dermis membranosa y areolada, con estriaciones en los segmentos abdominales. Setas dorsales de dos tipos, unas cónicas, grandes y redondeadas, de 7-12 (7-12) $\mu \mathrm{m}$ de longitud y 7-10 (7-10) $\mu \mathrm{m}$ de ancho, numerosas en toda la superficie, dispuestas en líneas transversas por segmento del cuerpo. Un segundo tipo de setas más angostas y de extremo agudo, de 12-14 (12-14) $\mu$ m de longitud, en los segmentos abdominales posteriores, intercaladas con las setas redondeadas, forman un grupo de 12 setas en el área media del segmento VII. Macroconductos ausentes. Microconductos aparentemente biloculares, de $3 \mu \mathrm{m}$ de longitud, numerosos, dispersos en márgenes y ubicados en el centro de cada areola en la superficie; microconductos de $14 \mu \mathrm{m}$ de longitud escasos en márgenes y dispersos en la superficie. Poros principalmente quinqueloculares de $5 \mu \mathrm{m}$ de diámetro y algunos triloculares, concentrados en la zona media de los últimos segmentos abdominales y en un grupo mediolateral de $40 \mu \mathrm{m}$ de longitud y ancho (la décima parte de la longitud del cuerpo), anterior en la región cefálica, distribuidos regularmente en el resto de la superficie. Lóbulos anales poco desarrollados, indicados por un grupo de seis setas de 12-17 (12-14) $\mu \mathrm{m}$ de longitud. Anillo anal y setas del anillo anal ausentes. Seta de 58 (48) $\mu \mathrm{m}$ a los lados de los lóbulos anales. Ano representado por una abertura membranosa de 19 (19) $\mu \mathrm{m}$ de ancho, rodeada por 12 setas de $12-$ 19 (12-19) $\mu \mathrm{m}$ de longitud cada una y dos setas mayores de 31 (24) $\mu \mathrm{m}$ de longitud, una a cada lado. Dos grupos de aproximadamente 15 poros en el área anal, corresponderían a dos invaginaciones alargadas o bolsas, características del género.

Margen. No definido.

Superficie ventral. Dermis membranosa. Setas de dos tipos, setas setosas de 7-13 (7-13) $\mu \mathrm{m}$ de longitud, con 18 setas (nueve en la zona media) en el segmento VII, en menor número, de 6-8 setas, en los segmentos abdominales posteriores. Setas cónicas redondeadas similares a las del dorso, de 7-12 (7-12) $\mu \mathrm{m}$ de longitud y 7-10 (7-10) $\mu \mathrm{m}$ de ancho, en áreas marginales y submarginales a lo largo del cuerpo, también laterales y submediales en segmentos abdominales y entre los espiráculos. Macroconductos ausentes. Microconductos escasos, dispersos en la superficie. Poros quinqueloculares de $5 \mu \mathrm{m}$ de diámetro, rodeando los espiráculos y en bandas transversas en abdomen, concentrados en zona media de últimos segmentos abdominales, dispersos en el resto de la superficie. Espiráculos con concavidad pequeña en el extremo anterior, cada una con aproximadamente 20-24 poros; del extremo interior parten numerosas tráqueas formando radios. Antenas unisegmentadas, de 36 (29) $\mu \mathrm{m}$ de longitud, con seis setas terminales. Clípeo-labro de 356 (317) $\mu \mathrm{m}$ de longitud. Labium de 89 (138) $\mu \mathrm{m}$ de longitud, con cuatro pares de setas y número desconocido de segmentos. Ancho de peritrema de espiráculos 48 (36) $\mu \mathrm{m}$. Patas ausentes. Vulva en segmento VII. 
Aspecto externo (Fig. 6). La hembra forma una cubierta esférica, la superficie exterior es de color negro; con un orificio pequeño de forma esférica (Hempel 1900).

\section{DISCUSIÓN}

Las cuatro especies de Apiococcus, al igual que Tectococcus ovatus, única especie del género, están presentes en Brasil y tienen como hospederos a Myrtaceae, en los cuales forman agallas. Carecen de poros o setas en el ano. Difieren por (caracteres de Tectococcus entre paréntesis): i) patas ausentes (presentes), ii) setas cónicas redondeadas en dorso y vientre (setas espinosas en dorso, flageladas en vientre), iii) macroconductos ausentes (presentes) y iv) lóbulos frontales ausentes (presentes).

El material de Apiococcus asperatus está en mal estado, se intentó realizar preparaciones microscópicas a partir del material seco conservado en las colecciones pero fue imposible recuperarlo, mediante hidratación y clarificación. Debido a que ese material está bajo el rotulo de "type" y es del año 1900 se decidió no perderlo intentando hacer preparaciones microscópicas para poder designar un Lectotipo. Sería oportuno realizar recolecciones de material provenientes de la localidad tipo, Brasil, São Paulo, Ipiranga sobre Myrtaceae, a fin de obtener material vivo de la especie para poder completar la redescripción de la misma, de acuerdo a los estándares actuales.

\section{AGRADECIMIENTOS}

A Carlos Eineker Lamas del Museu de Zoología, Universidad de São Paulo, Brasil, por el préstamo del material. A Andrea Saracho Bottero del INSUE - UNT, Argentina por la toma de las fotografías que ilustran las cubiertas externas.

\section{REFERENCIAS}

Claps, L.E. 1991. Morfología de estados inmaduros y adultos de Cornuaspis beckii (Newman, 1869) e Insulaspis gloverii (Packard, 1869) (Homoptera; Coccoidea; Diaspididae). Revista de la Sociedad Entomológica Argentina 49: 137-149.

Cockerell, T.D. 1899. Two new genera of Lecaniini Coccidae. The Entomologist 32: 12-13.

Ferris, G.F. 1957a. Notes on some little known genera of the Coccoidea (Homoptera). Microentomology 22: 59-79.

Ferris, G.F. 1957b. A review of the family Eriococcidae (Insecta: Coccoidea). Microentomology 22: 81-89.

Granara de Willink, M.C. 1990. Conociendo nuestra fauna 1. Superfamilia Coccoidea (Homopt. Sternor.). Serie Monográfica y Didáctica 6. Facultad de Ciencias Naturales e Instituto Miguel Lillo, Universidad Nacional de Tucumán, 43 p.

Hempel, A. 1900. As Coccidas Brazileiras. Revista do Museo Paulista 4 : 365-537.

Hempel, A. 1934. Descripcão de três especies novas, três gêneros novos e uma subfamília nova de Coccideos (Hemiptera, Homoptera). Revista de Entomologia 4: 139-147.

Hodgson C.J., Gonçalves, S.J., Miller, D.R. \& Isaias, R.S. 2004. A key to genera of Eriococcidae (Hemiptera: Coccoidea) from the Neotropical region and a revision of Pseudotectococcus Hempel (Eriococcidae), a gall inducing scale insect genus from Brazil, with a description of a new species. Lundiana 5: 51-72.

Hodgson, C. \& Miller, D. 2010. A review of the eriococcid genera (Hemiptera: Sternorrhyncha: Coccoidea) of South America. Zootaxa 2459: $1-101$.

Lepage, H.S. 1941. Descricão de um novo gênero e nova espécie de coccideo produtor de galhas (Homoptera-Coccoidea). Arquivos do Instituto Biologico 12: 141-145.

Miller, D.R. \& Gimpel, M.E. 2000. A Systematic Catalogue of the Eriococcidae (felt scales) (Hemiptera: Coccoidea) of the world. Andover, Intercept, 589 p.

Silva d'Araujo, G.A., Gonçalves, C.R., Galvão, G.M. \& Gonçalves, D.M. 1968. Quarto catalogo dos insetos que vivem nas plantas do Brasil. Parte II. Insetos, hospedeiros e inimigos naturais. V. 1, Rio de Janeiro, Ministerio da Cultura. $622 \mathrm{p}$. 